\title{
Development of a Piezo-driven Mechanical Stage Integrated Microdisplacement Sensor for Calibration of Displacements
}

\author{
Toshihiro Takeshita*, Takuma Iwasaki, Kota Harisaki, \\ Hideyuki Ando ${ }^{1}$, Eiji Higurashi ${ }^{2}$ and Renshi Sawada \\ Graduate School of Systems Life Sciences, Kyushu University, \\ 744 Motooka, Nishi-ku, Fukuoka 819-0395, Japan \\ 'Fuzzy Logic Systems Institute \\ 1-5 Hibikino, Wakamatsu-ku, Kitakyshu City, Fukuoka 808-0135, Japan \\ ${ }^{2}$ Research Center for Advanced Science and Technology, The University of Tokyo, \\ 4-6-1 Komaba, Meguro-ku, Tokyo 153-8904, Japan \\ (Received January 16, 2014; accepted June 19, 2014)
}

Key words: displacement sensor, optical sensor, piezoelectric actuator, position control, hysteresis

We fabricated a mechanical stage driven by a piezoelectric actuator and built in a microdisplacement sensor, which is used to decrease the effect of hysteresis and creep in the piezoelectric actuator. The displacement sensor consists of a vertical-cavity surface-emitting laser (VCSEL), eight two-dimensional monolithically integrated photodiodes (PDs), a frame, and a cover glass. The size of the sensor chip is $3.0 \mathrm{~mm}$ by $3.0 \mathrm{~mm}$ with a thickness of $0.7 \mathrm{~mm}$. The maximum variation in the position of the mechanical stage caused by hysteresis is $7.5 \mu \mathrm{m}$ without control when the movement range of the mechanical stage is $84.4 \mu \mathrm{m}$. By controlling the applied voltage using our microdisplacement sensor, we reduce the effect of the hysteresis, and the variation in position decreased to $1.9 \mu \mathrm{m}$.

\section{Introduction}

Piezoelectric actuators have many advantages, including a high generative force, low power consumption, fast response, and high resolution of displacement. The piezoelectric actuators are widely used in automobiles, robots, and medical machinery. Particularly, owing to the high resolution of displacement, piezoelectric actuators are widely used in many nanodriving and positioning systems. However, the piezoelectric actuator has hysteresis, which is nonlinear, and suffers from high thermal dependence. ${ }^{(1-4)}$ These factors all reduce the accuracy of the piezoelectric actuator. Therefore, displacement measurements

*Corresponding author: e-mail: e-mail: taskeshota@nano-micro.mech.kyushu-u.ac.jp 
of the piezoelectric actuator are required for position control. The displacement of measurement of piezoelectric actuators has already been reported. ${ }^{(5-7)}$ For example, the measurement and control of a piezo element displacement using strain gauges are reported in refs. 8 and 9. However, the strain gauge also suffers from thermal dependence similar to the piezo element, which varies its sensitivity on each device and limits the miniaturization of the strain gauge. Owing to the high-speed operation of a piezoelectric actuator, an optical displacement measurement method is suitable. Measurement of the displacement using an interferometer has already been reported. ${ }^{(10)}$ However, generally speaking, it requires a large space and a complex alignment. It can also be obstructive to apply the optical displacement measurement method to existing devices.

In our previous work, ${ }^{(11,12)}$ we studied and developed an optical displacement sensor. The principle of our sensor was laser triangulation. Geometry-based measurement methods such as this are preferred since they do not require a reference optical beam and have simple construction and high sensitivity. ${ }^{(13,14)}$ Moreover, our sensors do not require an optical fiber. Displacement sensors using an optical fiber can measure in small spaces and are also resistant to electric noise. ${ }^{(15,16)}$ However, the sensor structure becomes quite complex, and the sensor size increases in comparison with a sensor that does not use an optical fiber. A simple construction will enable a marked miniaturization. A vertical-cavity surface-emitting laser (VCSEL) is used in this work to form our displacement sensor. It is a simple optical system with many advantages such as low energy consumption and low thermal dependence. ${ }^{(17)}$ The primary features of our sensor are small size $\left(3.0 \times 3.0 \mathrm{~mm}^{2}\right.$ and $0.7 \mathrm{~mm}$ thick $)$, one axis linear motion and inclination around two axes measurement, simple structure, small number of components, and low power consumption.

In this work, we fabricated a mechanical stage driven by a piezoelectric actuator. Moreover, we measured and controlled the displacement using our microdisplacement sensor to solve the problems of hysteresis, creep, and nonlinearity of the piezoelectric actuator. Since the displacement sensor is small and does not require complex alignment, it is expected that it could be applied to existing products with minimal interference.

\section{Materials and Methods}

\section{$2.1 \quad$ Piezo-actuated mechanical stage}

Figure 1 shows the mechanical stage that we fabricated. The stage is made up of four components: piezoelectric actuator, microdisplacement sensor that we developed, component 1 , and component 2. The piezoelectric actuator (AE0203D44H40F, NECTokin) is sandwiched by component 1 . The maximum displacement of the piezo element is $42.0 \mu \mathrm{m}$ when the applied voltage is $150 \mathrm{~V}$. An external mirror, which is needed to measure the displacement, is attached to the ceiling of component 2. The mirror was fabricated by sputtering chrome to the silicon substrate and is $3 \mathrm{~mm}^{2}$. The position of the displacement sensor is adjusted to place it under the external mirror without inclination using three clinchers. The distance from the sensor surface to the external mirror is 3000 $\mu \mathrm{m}$. 


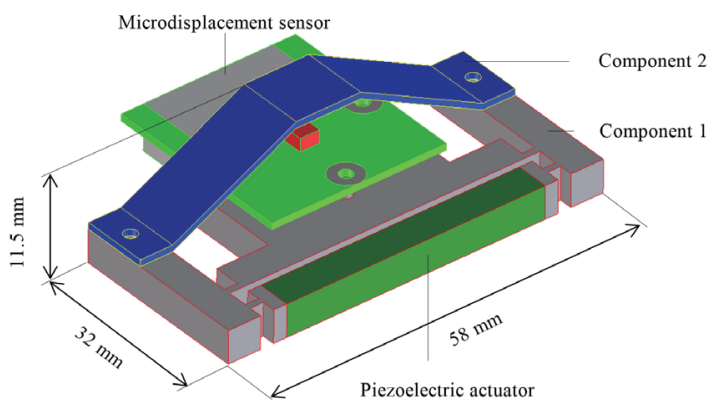

(a)

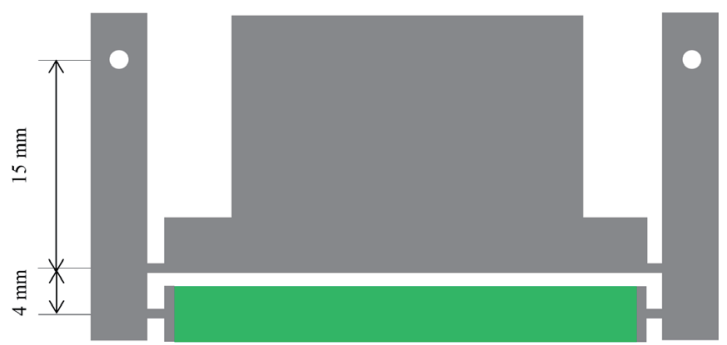

(b)

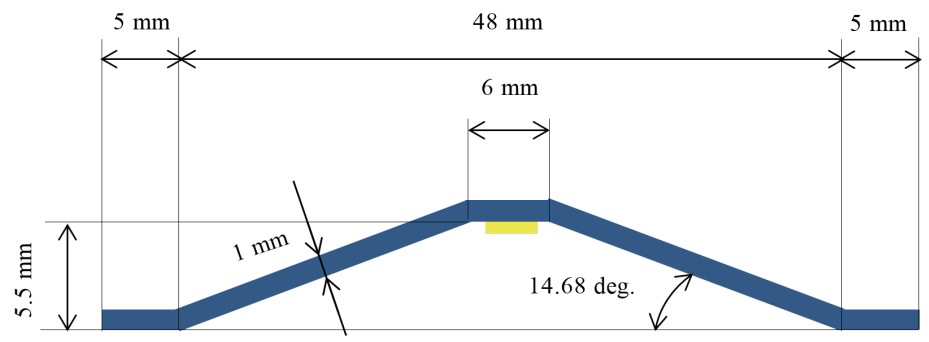

(c)

Fig. 1. (Color online) Piezo-actuated mechanical stage structure and design: (a) structure, (b) design of component 1 , and (c) design of component 2 .

The materials of components 1 and 2 are stainless steel. The displacement of the piezoelectric actuator is amplified by components 1 and 2 . The piezoelectric actuator and component 1 are attached using an adhesion bond. Components 1 and 2 are screwed and attached using an adhesion bond to eliminate the backlash between each component.

\subsection{Microdisplacement sensor}

Figure 2 shows the structure of the displacement sensor that we developed. A VCSEL chip is bonded to the center of the silicon wafer. The detection photodiodes (PDs), PD-A1 - PD-D3, and the monitoring PD are monolithically integrated to the silicon wafer base. These detection PDs are arranged concentrically around the VCSEL. An external mirror is attached to a measuring object. The light emitted from the VCSEL is reflected off the external mirror and enters the detection PDs. The VCSEL and PDs are wire bonded on the assembled substrate. There are primal amplifiers on the substrate to amplify the PD signals. A frame is then bonded onto the assembled substrate.

The basic measurement concept of the sensor is described in Fig. 3. A fraction of the laser beam transmitted through the cover glass reflects off an external mirror and back toward the 12 detection PDs. When these external objects are linearly translated, the 

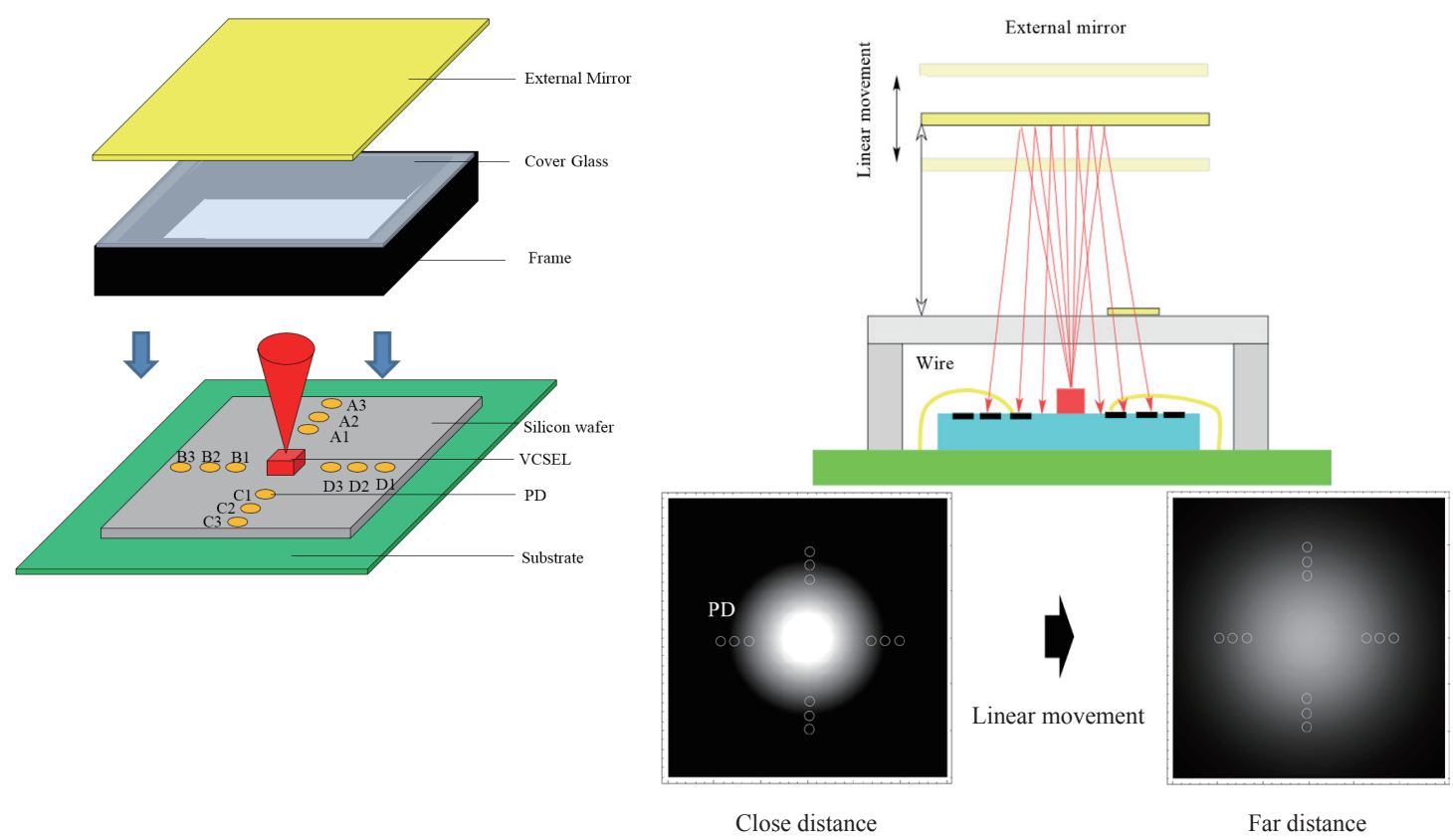

Fig. 2 (left). (Color online) Structure of microdisplacement sensor.

Fig. 3 (right). (Color online) Principle of microdisplacement sensor.

diameter and intensity of the light beam are altered. The sensor can measure the linear displacement of the external mirror from these changes. The measurement range of the device is the region in which the signals from the detection PDs vary proportionally with the linear movement of the external mirror.

Figure 4 shows the design of the sensor chip. These PDs are rounded and are composed of PN photodiodes. The inner PDs, middle PDs, and outer PDs are arranged concentrically around the VCSEL, with radii $r_{\mathrm{i}}, r_{\mathrm{c}}$, and $r_{\mathrm{o}}$, respectively. $r_{\mathrm{c}}$ denotes the distance from the VCSEL to the monitoring PD. The size of the sensor chip is $3 \mathrm{~mm}$ by $3 \mathrm{~mm}$. In this figure, $z_{\mathrm{m}}$ is the gap between the surface of the cover glass and the object to be measured. The sensor thickness is $1600 \mu \mathrm{m}$, including the frame and cover glass.

We first calculate the theoretical output of PDs using Mathematica (Wolfram). Figure 5 shows the theoretical result when the mirror moves from the sensor surface linearly. In this figure, $S_{\text {in }}, S_{\text {middle }}$, and $S_{\text {out }}$ are the sums of the outputs of the inner PDs, middle PDs, and outer PDs, respectively, and defined using the following equations. Pn means optical power on PD- $n$.

$$
\begin{gathered}
S_{\text {in }}=P A 1+P B 1+P C 1+P D 1 \\
S_{\text {middle }}=P A 2+P B 2+P C 2+P D 2 \\
S_{\text {out }}=P A 3+P B 3+P C 3+P D 3
\end{gathered}
$$




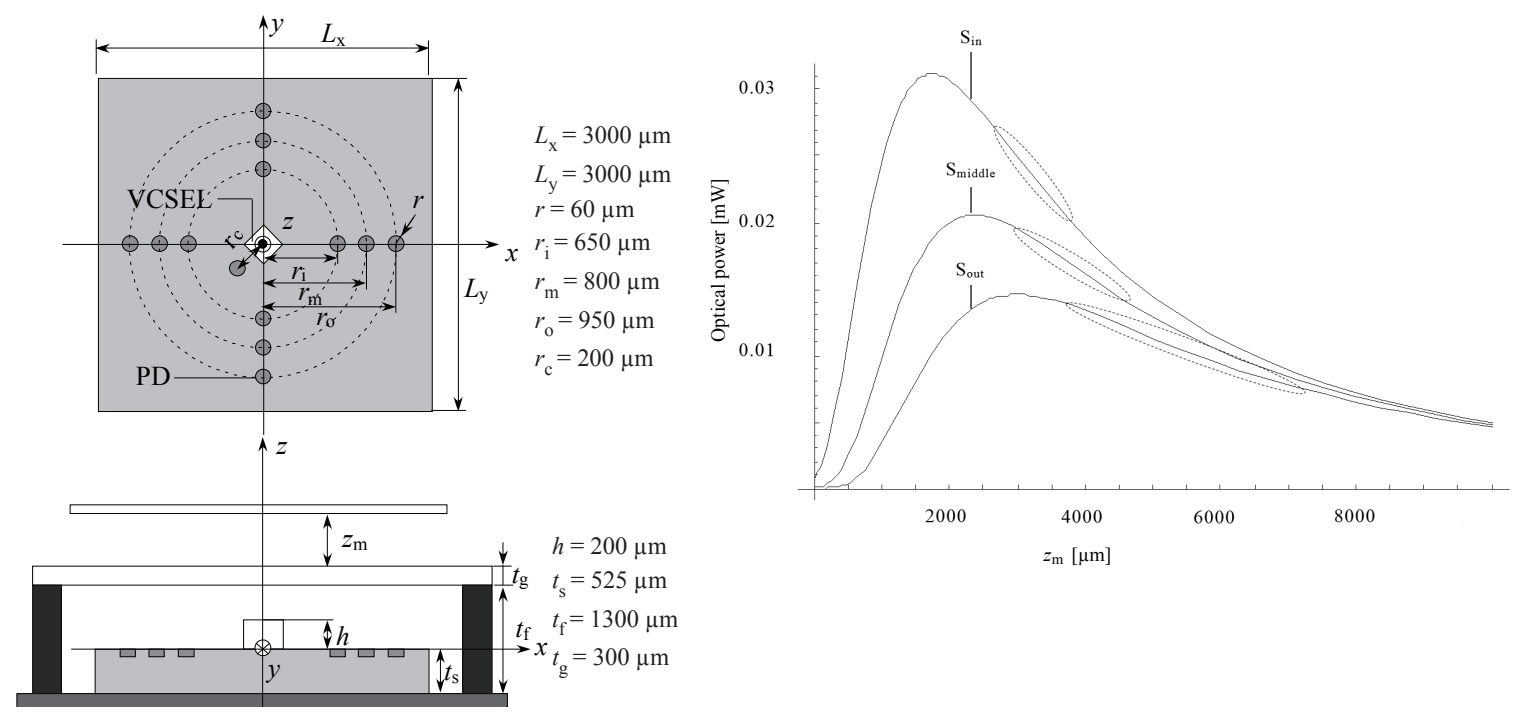

Fig. 4 (left). Design of microdisplacement sensor.

Fig. 5 (right). Theoretical result.

It is expected that the use of the 4 PDs removes minor variations in the inclination of the subject being measured. $S_{\text {in }}$ is suited for high-resolution measurements since its output voltage is larger. In comparison, $S_{\text {out }}$ is suited for long-range measurements since the linear region of its output signal is longer. In our magnification application, the displacement stage is designed to be in the range of hundreds of micrometers and requires accurate control. Therefore, we used the $S_{\text {in }}$ value for this application. We can determine the linear signal when the distance from the sensor surface to the external mirror is between 2500 and $3500 \mu \mathrm{m}$.

A SEM image of our displacement sensor is shown in Fig. 6. In this figure, the cover glass is removed to take the picture.

The VCSEL specifications are as follows: single mode and beam divergence of $16.9 \mathrm{deg}$. $\left(1 / e^{2}\right)$, a wavelength of $850 \mathrm{~nm}$, and a typical optical power of $1.92 \mathrm{~mW}$. The VCSEL is bonded on the sensor chip using Ag paste (XA-910, Fujikura Kasei Co., Ltd.), and the chip is heated under the temperature limit of the VCSEL. The PDs and VCSEL are wired with Au ( $20 \mu \mathrm{m}$ diameter) to the amplifier substrate by wire bonding. To arrange the monitoring PD close to the VCSEL, the VCSEL is rotated $45^{\circ}$ in the direction of the sensor chip. The frame and cover glass are attached by an adhesion bond. The sensor has the following features: the size of the sensor chip is small, the power consumption is by the VCSEL, and the measurement range and resolution can be adapted by selecting the PD signals and high response speed $(240 \mu \mathrm{s})$. 


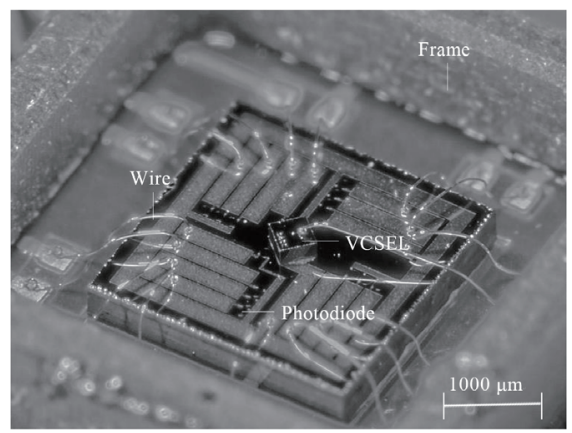

Fig. 6. Picture of microdisplacement sensor.

\section{Methods}

\subsection{Experimental setup}

The experimental setup for this section is shown in Fig. 7. The piezo element has a voltage applied from the PC through the piezo-driven amplifier. The maximum output voltage from the PC is $5.0 \mathrm{~V}$ and the output voltage is controlled by LabVIEW ${ }^{\mathrm{TM}}$ (National Instrument). The gain of the amplifier is 30 ; therefore, the maximum applied voltage on the piezoelectric actuator is $150 \mathrm{~V}$. The displacement at the measurement site is also measured using the laser displacement sensor (LK-G15, Keyence).

The output signals of the displacement sensor and laser displacement sensor are filtered through a low-pass filter $(1000 \mathrm{~Hz})$ on LabVIEW ${ }^{\mathrm{TM}}$ and measured using LabVIEW $^{\mathrm{TM}}$. We adjusted the PD output by multiplying with a coefficient calculated in advance. This was to remove the variability in the sensitivity of each PD.

The mechanical stage is shown in Fig. 8. The laser displacement sensor (LKG15, Keyence) allows us to measure the displacement of the external mirror within an accuracy of $0.01 \mu \mathrm{m}$. The resolution of this laser displacement sensor is therefore $0.1 \mu \mathrm{m}$. The effect of hysteresis must be removed to accurately drive the piezoelectric actuator. However, from Fig. 6, a commercialized laser displacement sensor is too large to insert in the mechanical stage. By comparison, our displacement sensor is seen to be very small.

\subsection{Experiment 1 and result}

First, to determine the hysteresis of this mechanical stage driven by a peizoelectric actuator, we measured the displacement of the ceiling when the amplified voltage of the piezoelectric continuously changes from $0 \rightarrow 150 \rightarrow 0 \mathrm{~V}$. The applied voltage has a triangular wave shape and a frequency of $0.05 \mathrm{~Hz}$. The experiment is conducted on a vibration-isolated table at room temperature.

The result of this experiment is described in Fig. 9. The maximum difference in displacement is $7.5 \mu \mathrm{m}$ when the applied voltage is $49.8 \mathrm{~V}$, as can be seen in Fig. 9(a). The maximum displacement is $84.4 \mu \mathrm{m}$ and the magnification ratio is 2.01 when the 


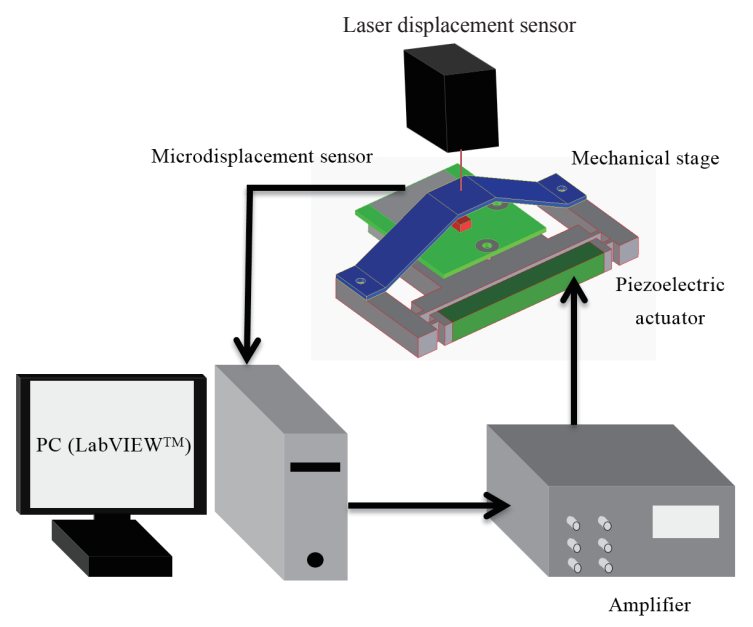

Fig. 7. (Color online) Experimental system and setup.

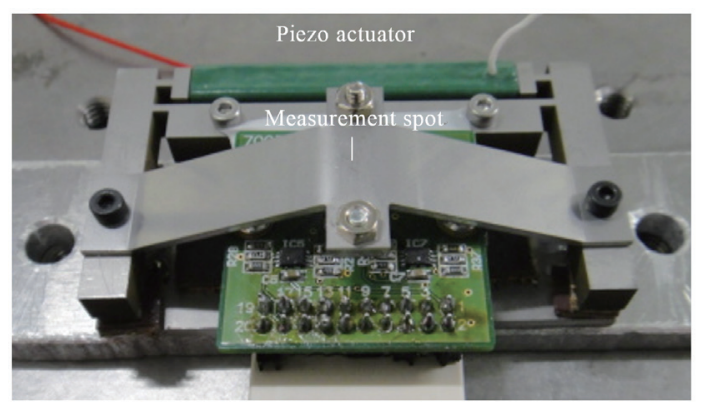

(a)

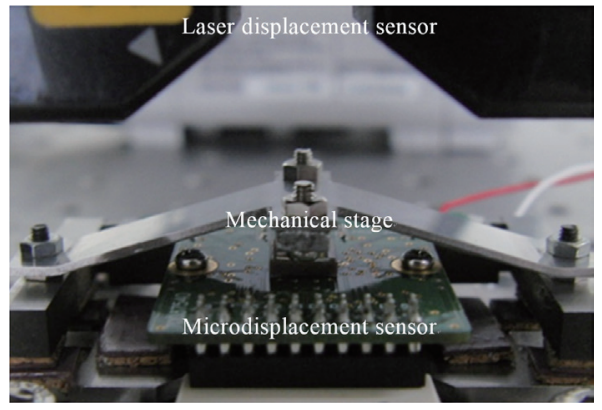

(b)

Fig. 8. (Color online) Picture of piezo-actuated mechanical stage.

applied voltage is $150 \mathrm{~V}$. Figure 9(b) shows the output of the microdisplacement sensor. From this result, the sensor can detect hysteresis equally as well as the laser displacement sensor. Figure 9(c) shows the relationship between the displacement and the output of $S_{\text {in }}$. From this result, we can calculate the linear signal of $S_{\text {in }}$. The slope is $2.33 \mathrm{mV} / \mu \mathrm{m}$ and the correlation coefficient is 0.9996 . From this result, the electrical resolution is 1.33 $\mu \mathrm{m}$, which is calculated using the full width at half maximum (FWHM) of this signal when the measurement range is $84.4 \mu \mathrm{m}$.

\subsection{Experiment 2 and result}

Next, we control the displacement of the mechanical stage using the microdisplacement sensor. First, we chose an initial position. We set the output voltage at $0 \mathrm{~V}$. Thus, the applied voltage on the piezoelectric actuator is $0 \mathrm{~V}$. Then, the output signal of the displacement sensor entered LabVIEW ${ }^{\mathrm{TM}}$. This is the initial setup. Next, 


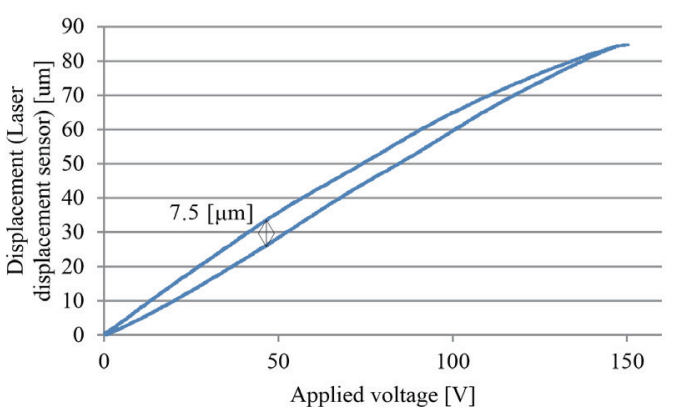

(a)

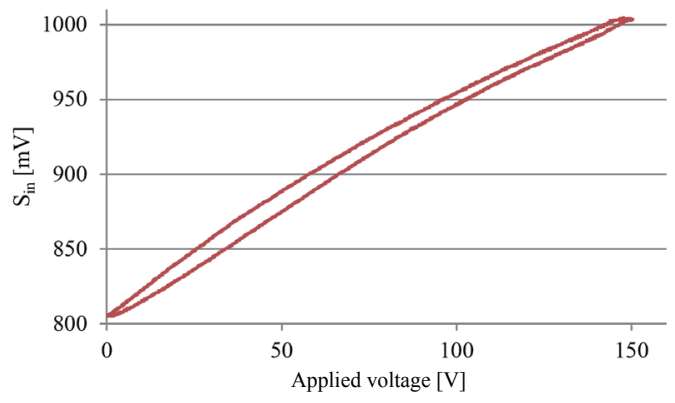

(b)

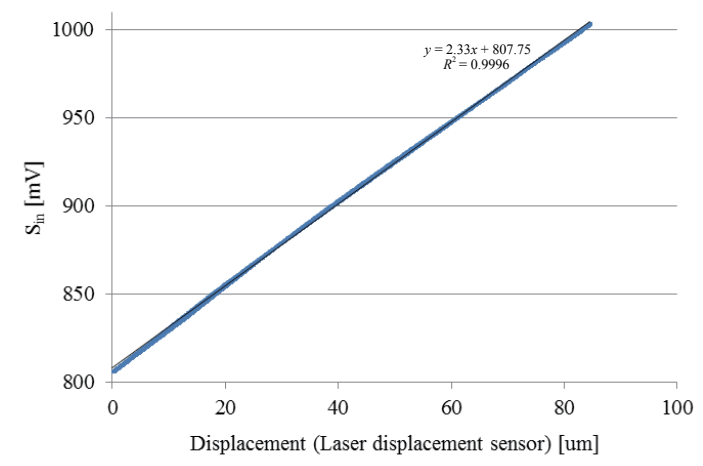

(c)

Fig. 9. (Color online) Result of experiment 1. (a) Laser displacement sensor output, (b) microdisplacement sensor output, and (c) linearity of displacement sensor.

we input the displacement that we wanted to move in LabVIEW ${ }^{\mathrm{TM}}$. When we wanted to elevate the measurement site, the applied voltage is decreased. In contrast, to lower the measurement site, the applied voltage is increased. We determine the target value in the change in the displacement sensor output using

$$
y=a x+b .
$$

Here, $x, y, a$, and $b$, are the target value, slope of the signal in the measurement range, intercept, and displacement value we wished to move, respectively. From the result shown in Fig. 9(c), $a=2.33 \mathrm{mV} / \mu \mathrm{m}$ and $b=807.75 \mathrm{mV}$. LabVIEW ${ }^{\mathrm{TM}}$ was used to adjust the applied voltage every $1 \mathrm{~ms}$ until the output of the displacement sensor reached the desired target value. We then measured the displacement at the displacement site using the laser displacement sensor. We changed the input value from 0 to $80 \mu \mathrm{m}$ and then from 80 to $0 \mu \mathrm{m}$ in $10 \mu \mathrm{m}$ increments and measured the displacement. 
Figure 10 shows the experimental results, where measurements were taken only after the displacement stabilized. Figure 10 shows the signals of the laser displacement sensor and $S_{\text {in }}$ in this experiment (in this figure, for example, the target values are 50 and 60 $\mu \mathrm{m})$. The changes in each signal after the target values are changed are shown. When the signal of $S_{\text {in }}$ reaches the target value calculated using eq. (4), the signal becomes constant. The signal of the laser displacement sensor is the same as that of $S_{\text {in }}$.

The result of this experiment is shown in Fig. 11. It can be seen that the effect of the hysteresis decreases without control. The maximum variation is $1.9 \mu \mathrm{m}$ when the input value is $50 \mu \mathrm{m}$. In comparison with $7.5 \mu \mathrm{m}$, which is the maximum difference caused by the hysteresis, we can decrease its effect.

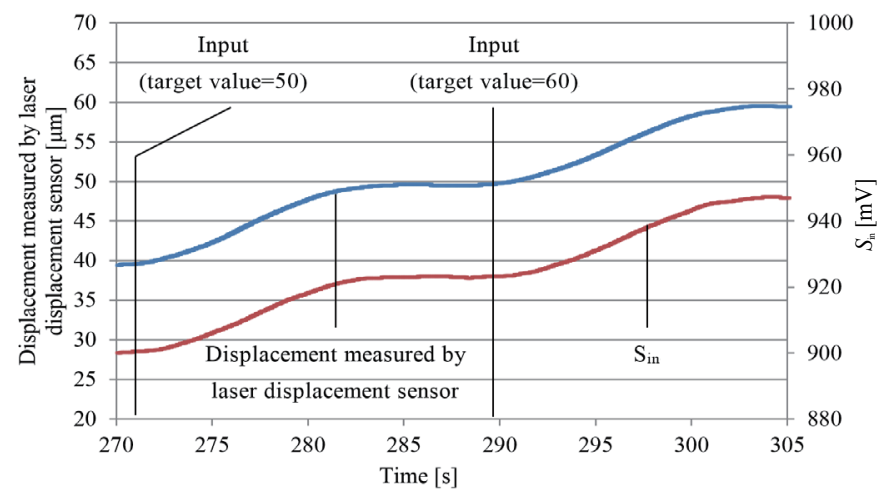

Fig. 10. (Color online) Example of controlled mechanical stage position.

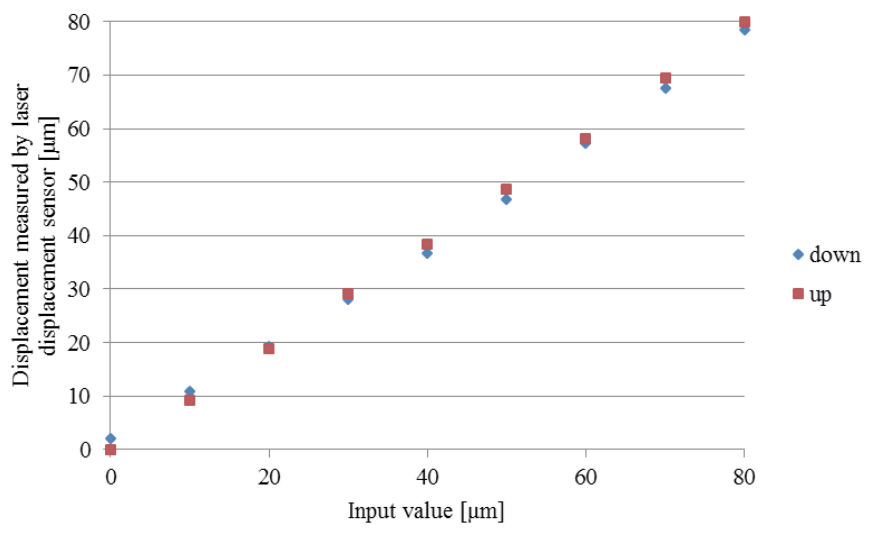

Fig. 11. (Color online) Result of experiment 2. 


\section{Discussion}

The maximum variation between the up and down mechanical stage is $1.9 \mu \mathrm{m}$ when the target value is $50 \mu \mathrm{m}$. In comparison with $7.5 \mu \mathrm{m}$, which is the maximum difference caused by hysteresis without control, we can decrease the effect of the hysteresis. However, there are some differences between the target value and the signals of the laser displacement sensor. The maximum value is $3.3 \mu \mathrm{m}$ when down the mechanical stage and the target value is $60 \mu \mathrm{m}$. A major cause is noise in the sensor. From the result shown in Fig. 9(c), the electrical resolution of the microdisplacement sensor is 1.33 $\mu \mathrm{m}$. Therefore, the effect of noise is stronger than expected. It is necessary to achieve a higher resolution for more accurate control. The PD outputs are amplified by OPAMP on the PCB substrate in the Au wires. The wires and circuit on the PCB substrate can cause substantial electrical noise. In the future, we will integrate preamplifiers on the sensor chip to decrease the amount of noise. Moreover, with the integration of preamplifiers, the PCB substrate becomes obsolete, and the miniaturization of the microdisplacement sensor is expected. Another possible cause is the inclination of the measurement spot. The difference in inclination is dependent on the applied voltage. However, our sensor is expected to eliminate the effect of inclination by calculating each PD output. ${ }^{(18)}$ Moreover, using two piezoelectric actuators, it is possible to control the stage position without inclination. The sensor can measure the linear movement in addition to two axial inclination angles. In this paper, we reported a linear displacement mechanical stage and controlled the linear displacement using our sensor.

In the future, we plan to fabricate a linear displacement and two axial inclination mechanical stages using a number of piezoelectric actuators. Our novel sensor will be used for control.

\section{Conclusions}

We fabricated a mechanical stage for a piezoelectric actuator. To decrease the effect of hysteresis, we also controlled the displacement of the piezoelectric actuator using a novel microdisplacement sensor. The expansion ratio of the mechanical stage is 2.01 and the maximum difference in position is $7.5 \mu \mathrm{m}$. The microdisplacement sensor is small enough to insert in confined spaces. The applied voltage of the piezoelectric actuator was controlled every $1 \mathrm{~ms}$ to adjust the displacement to a desired value regardless of whether the measurement spot went up or down. Our experiments demonstrated that the effect of hysteresis was reduced. However, the effect of electrical noise from the wires and circuit at the preamplifier substrate limits the maximum value of this structure to $1.9 \mu \mathrm{m}$. In the future, we intend to design a new sensor chip on which we integrate the preamplifiers to further miniaturize the microdisplacement sensor, and also increase its resolution. The optical elements, which are the photodiodes and VCSEL, also suffer from thermal dependence. This can limit the accuracy of the displacement sensor. Therefore, we will also integrate a thermal transistor to measure the temperature of the chip and control. 


\section{References}

1 S. Jung and S. Woo: Precis. Eng. 16 (1994) 49.

2 C. V. Newcomb and I. Flinn: Electron. Lett. 18 (1982) 442.

3 A. Ochi, S. Takahashi and S. Tagami: Jpn. J. Appl. Phys. 24 (1985) 209.

4 C. Jer Lin and S. Ren Yang: Mechatronics 16 (2006) 417.

5 S. Hung Chang, C. Kai Tseng and H. Chan Chien: IEEE Trans. Ultrason. Ferroelectr. Freq. Control 46 (1999) 906.

6 A. E. Holman, W. Chr. Heerens and F. Tuinstra: Sens. Actuators, A 36 (1993) 37.

7 Y. Tien Liu and H. Toshiro: IEEE/ASME Trans. Mechatron. 6 (2001) 467.

8 Y. Yu, B. Song, R. Chen and Y. Ge: Proc. Intelligent Robots and Systems (IROS) 2010 (IEEE/ RSJ) 2010, pp. 2618-2624.

9 Y. Yu, B. Song and Y. Ge: Proc. Intelligent Robots and Systems (IROS) 2009 (IEEE/RSJ) 2009, pp. 3290-3296.

10 S. Hung Chang, C. Kai Tseng and H. Chan Chien: IEEE Trans. Ultrason. Ferroelectr. Freq. Control 46 (1999) 897.

11 I. Ishikawa, R. Sawada, E. Higurashi, S. Sanada and D. Chino: Sens. Actuators, A 138 (2007) 269.

12 T. Iwasaki, T. Takeshita, Y. Arinaga and R. Sawada: Sens. Mater. 25 (2013) 601.

13 J. Clark, A. M. Wallace and G. L. Pronzato: IEEE Trans. Robot. Autom. 14 (1998) 60.

14 M. Caccia: IEEE J. Oceanic Eng. 31 (2006) 711.

15 T. Wang, S. Zheng and Z. Yang: Sens. Actuators, A 69 (1998) 134.

16 J. Brandao Faria: IEEE Trans. Instrum. Meas. 47 (1998) 742.

17 I. Kenichi: IEEE J. Sel. Top. Quant. Electron. 6 (2000) 1201.

18 T. Takeshita, Y. Peng, N. Morita, H. Ando, E. Higurashi and R. Sawada: Joint Int. IMEKO Symp, 2011, urn:nbn:de:gbv:ilml-2011imeko-015:6. 